\title{
2. Liste der praesides und comites Isauriae
}

\begin{tabular}{|c|c|c|c|c|}
\hline Jahr & $\begin{array}{l}\text { Name und } \\
\text { Rang }\end{array}$ & $\begin{array}{l}\text { literarische } \\
\text { Quelle }\end{array}$ & inschriftliche Quelle & Sekundärliteratur \\
\hline $\begin{array}{l}\text { Ende 3. / } \\
\text { Anfang 4. } \\
\text { Jh. }\end{array}$ & $\begin{array}{l}\text { Amyntas, } \\
\text { praeses } \\
\text { Isauriae }\end{array}$ & $\%$ & 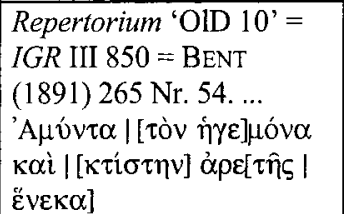 & $\begin{array}{l}\text { PLRE I } 58 \text { s.n. } \\
\text { 'Amyntas'; PIR I } \\
571 .\end{array}$ \\
\hline $306-309$ & $\begin{array}{l}\text { Lucilius } \\
\text { Crispus, } \\
\text { praeses } \\
\text { Isauriae }\end{array}$ & $\%$ & $\begin{array}{l}\text { Repertorium } 374 \text { 'Sel } \\
140 \text { ' = AÉ 1978, 814 = } \\
\text { SAHIN (1991) 152f. Nr. 2c } \\
=\text { DAGRON / FEISSEL } \\
\text { (1987) } 20 \mathrm{Nr} .2 \text {. = BM II } \\
\text { 196f. Nr. 217; } \\
\text { Repertorium 'Sel 141' = } \\
\text { AÉ 1978, 815 = DAGRON / } \\
\text { FEISSEL (1987) } 21 \mathrm{Nr} .3= \\
\text { ŞAHIN (1991) } 152 \mathrm{Nr} .2 \mathrm{~b}\end{array}$ & $\begin{array}{l}\text { PLRE I } 233 \text { s.n. } \\
\text { 'Crispus 5' (nur als } \\
\text { vicarius); ŞAHIN } \\
\text { (1991) 15If. }\end{array}$ \\
\hline $309-313$ & $\begin{array}{l}\text { Flavius } \\
\text { Severianus, } \\
\text { praeses } \\
\text { Isauriae }\end{array}$ & $\%$ & $\begin{array}{l}\text { Repertorium } 377 \text { 'Sel } \\
\text { 150'= ŞAHIN (1991) 150; } \\
\text { Repertorium } 373 \text { 'Sel } \\
\text { 136' = ŞAHIN (1991) 152, } \\
\text { Nr. 2a = BM II 196f., Nr. } \\
\text { 217; ILS 660; } \\
\text { Repertorium 277, 'Kry } \\
501 \text { ' } \\
=\text { MAMA III Nr. } 199 \mathrm{~b}\end{array}$ & $\begin{array}{l}\text { ECK (1973); } \\
\text { MARTINDALE (1980) } \\
\text { 493; MATHISEN } \\
\text { (1987) 13; SAHIN } \\
\text { (1991) 151f. }\end{array}$ \\
\hline $\begin{array}{l}\text { ca. } \\
313-324\end{array}$ & $\begin{array}{l}\text { Aurelius } \\
\text { Fortunatus, } \\
\text { praeses } \\
\text { Isauriae }\end{array}$ & . & $\begin{array}{l}\text { Repertorium } 375 \text { 'Sel } \\
\text { 142a' }=A \dot{E} 1978,816= \\
\text { ŞAHIN }(1991) 153 \text { Nr. } 2 \mathrm{~d} \\
=\text { DAGRON / FEISSEL } \\
\text { (1987) } 22 \mathrm{Nr} .4 ; \\
\text { Repertorium } 382 \text { 'SIT 22' } \\
=\text { BM II } 154 \mathrm{f} \text {. Nr. } 157 .\end{array}$ & ŞAHIN (1991). \\
\hline 354 & $\begin{array}{l}\text { Castricius, } \\
\text { comes } \\
\text { Isauriae }\end{array}$ & $\begin{array}{l}\text { Amm. XIV 2, } \\
\text { 14; Lib. ep. } \\
426 .\end{array}$ & $\%$ & $\begin{array}{l}\text { PLRE I } 186 \text { s.n. } \\
\text { 'Castricius 1'; SEECK } \\
\text { (1906) 103f. }\end{array}$ \\
\hline $\begin{array}{ll}\text { ca. } & 355- \\
359 & \end{array}$ & $\begin{array}{l}\text { Aurelius } \\
\text { Iustus, comes } \\
\text { et praeses } \\
\text { Isauriae }\end{array}$ & $\%$ & $\begin{array}{l}\text { Repertorium 82, 'Eir l'= } \\
\text { BM II 206, Nr. } 231=\text { AÉ } \\
\text { 1974, } 644 \text { (Eirenopolis) }\end{array}$ & $\begin{array}{l}\text { ARCE }(1973) ; \\
\text { MARTINDALE }(1980) \\
\text { 487; MATHISEN } \\
(1987) 13 .\end{array}$ \\
\hline
\end{tabular}




\begin{tabular}{|c|c|c|c|c|}
\hline $359--362$ & $\begin{array}{l}\text { Bassidius } \\
\text { Lauricius, } \mathrm{vc}, \\
\text { comes et } \\
\text { praeses } \\
\text { Isauriae }\end{array}$ & $\begin{array}{l}\text { Amm. XIX 13, } \\
2 \text {; Socr. h.e. II } \\
39 ; \text { Sozom. h.e. } \\
\text { IV } 22,2 \text {; Jul. } \\
\text { ep. } 80 \text {; Lib. ep. } \\
585 ; \\
\text { Epiphanius, } \\
\text { adversus Hae- } \\
\text { reses } 73,25,3 \text {. }\end{array}$ & $I L S 740=$ CIL III 6733. & $\begin{array}{l}\text { PLRE I } 497 \text { s.n. } \\
\text { 'Bassidius Lauricius'; } \\
\text { RouGÉ (1966); } \\
\text { BARNES (1998) } 92 \text { u. } \\
\text { Anm. 76; SCHARF } \\
(1990) .\end{array}$ \\
\hline $363-365$ & $\begin{array}{l}\text { Olympius } \\
\text { Palladius, } \\
\text { praeses } \\
\text { Isauriae }\end{array}$ & $\begin{array}{l}\text { Lib.ep. } 689 . \\
837.1133 . \\
1151-1153 . \\
1168.1169 . \\
1204.1207 . \\
1238.1249 . \\
1438.1470 . \\
1486 .\end{array}$ &. & $\begin{array}{l}\text { PLRE I 662 s.n. } \\
\text { 'Olympius Palladius } \\
\text { 18'.258 s.n. } \\
\text { 'Dionysius 6'; SEECK } \\
\text { (1906) 119. 121. } 228 \\
\text { 'Palladius V'. }\end{array}$ \\
\hline $\begin{array}{l}366-367 \\
(?)\end{array}$ & $\begin{array}{l}\text { Flavius } \\
\text { Uranius, vc, } \\
\text { praeses } \\
\text { Isauriae } \\
(\lambda \alpha \mu \pi \rho o ́ \tau \alpha \tau o \\
\text { só } \rho \chi \omega \vee)\end{array}$ & $\%$ & $\begin{array}{l}\text { Repertorium } 170, \text { 'Krs 1' } \\
=\text { CIG 4430; LANGLOIS } \\
(1861) 194 \text {; BENT (1891) } \\
258, \mathrm{Nr} .31 ; \text { OGIS II } \\
580 ; \\
M A M A \text { III } 102, \mathrm{Nr} .3\end{array}$ & $\begin{array}{l}\text { PLRE I } 982 \text { s.n. 'Fl. } \\
\text { Vranius 5'; MAMA III } \\
\text { 102-107. }\end{array}$ \\
\hline $\begin{array}{l}368-374, \\
375-377 \\
(?)\end{array}$ & $\begin{array}{l}\text { Flavius } \\
\text { Saturninus, } \\
\text { comes rei } \\
\text { militaris aus } \\
\text { Antiochia; } \\
\text { wahrscheinl. } \\
\text { später comes } \\
\text { et praeses } \\
\text { Isauriae } \\
\end{array}$ & 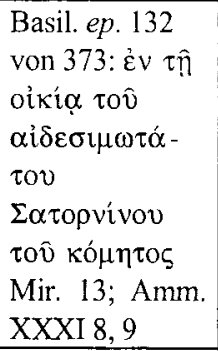 & $\%$ & $\begin{array}{l}\text { PLRE I 807f., s.n. } \\
\text { 'Saturninus 10'; } \\
\text { DAGRON (1978) 117f.; } \\
\text { LENSKI (1999 b) } \\
315 \mathrm{ff} .\end{array}$ \\
\hline $\begin{array}{l}378-379 \\
(?)\end{array}$ & $\begin{array}{l}\text { Valerius } \\
\text { Valentinianus, } \\
\text { praeses } \\
\text { Isauriae } \\
\end{array}$ & $\%$ & $\begin{array}{l}\text { HALL }(1972) ; A \vec{E} 1972 \text {, } \\
659 .\end{array}$ & $\begin{array}{l}\text { HALL (1972); } \\
\text { MARTINDALE (1980) } \\
\text { 495; } \\
\text { MATHISEN (1987) } 13 .\end{array}$ \\
\hline $\begin{array}{l}380-381 \\
(?)\end{array}$ & $\begin{array}{l}\text { Natalis, } \\
\text { dux Isauriae }\end{array}$ & $\begin{array}{l}C T h \text { IX } 27,3= \\
C J \operatorname{IX~} 27,1\end{array}$ & $\%$ & $\begin{array}{l}\text { PLRE I } 617 \text { s.n. } \\
\text { 'Natalis'; ALFÖLDI- } \\
\text { RoSENBAUM (1972) } \\
184 .\end{array}$ \\
\hline
\end{tabular}




\begin{tabular}{|c|c|c|c|c|}
\hline $382-383$ & $\begin{array}{l}\text { Matronianus, } \\
\text { dux et praeses } \\
\text { Isauriae }\end{array}$ & $\begin{array}{l}C T h \text { IX 27, } 3= \\
C J \operatorname{IX~27,1}\end{array}$ & $\begin{array}{l}\text { Repertorium 28, 'Anm } \\
\text { 16' = ALFÖLDI- } \\
\text { RoSENBAUM (1972), } \\
\text { korr. JONES (1972); } \\
\text { MERKELBACH (1973) }\end{array}$ & $\begin{array}{l}\text { PLRE I } 568 \text { s.n. } \\
\text { 'Matronianus 2'; } \\
\text { SEECK (1919) 259; } \\
\text { Wilhelm ENSSLIN, s.v. } \\
\text { 'Matronianus 2', RE } \\
\text { XIV (1930) 2309; } \\
\text { Ders., s.v. 'Natalis 3', } \\
\text { RE XVI } 2 \text { (1935) } \\
\text { 1799; ALFÖLDI- } \\
\text { RoSENBAUM (1972); } \\
\text { JONES (1972); } \\
\text { MERKELBACH (1973); } \\
\text { SCHARF (1990); HILL } \\
\text { (1985); HILD / } \\
\text { HELLENKEMPER } \\
\text { (1986) 82 ff.; WOODS } \\
\text { (1998) 110, Anm. 6. }\end{array}$ \\
\hline $392(?)$ & $\begin{array}{l}\text { Demonicus, } \\
\text { comes } \\
\text { Isauriae (?) }\end{array}$ & $\begin{array}{l}\text { Lib. ep. } 1054 . \\
1055\end{array}$ & $\%$ & $\begin{array}{l}\text { PLRE I } 249 \text { s.n. } \\
\text { 'Demonicus'; SEECK } \\
\text { (1906) 120, 443; } \\
\text { SCHARF (1993) } 218 \mathrm{f} .\end{array}$ \\
\hline $\begin{array}{l}(396- \\
400)\end{array}$ & \begin{tabular}{|l|} 
Flavius \\
Fravitta, nicht \\
comes \\
Isauriae, \\
sondern \\
magister \\
militum per \\
Orientem \\
\end{tabular} & 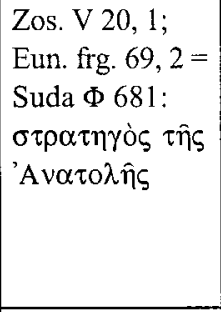 & $\%$ & $\begin{array}{l}\text { PLRE I 372f. s.n. } \\
\text { 'Flavius Fravitta'; } \\
\text { DEMANDT (1970) } \\
\text { 736; CAMERON / } \\
\text { LONG (1993) 224; } \\
\text { BURNS (1994) 173; } \\
\text { WOODS (1998) 117. }\end{array}$ \\
\hline $\begin{array}{l}\text { ca. } 400- \\
404\end{array}$ & \begin{tabular}{|l|} 
Flavius \\
Leontius, \\
comes primi \\
ordinis et dux \\
Isauriae \\
\end{tabular} & $\%$ & 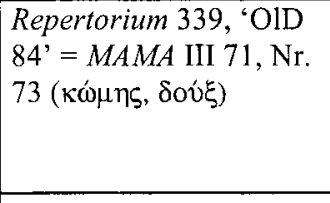 & $\begin{array}{l}\text { PLRE II } 674 \text { s.n. 'Fl. } \\
\text { Leontius } 28 \text { ' ,395- } \\
\text { 402“; TIB 5,1 } 240 \text { s.v. } \\
\text { 'Diokaisareia'. }\end{array}$ \\
\hline $404(?)$ & $\begin{array}{l}\text { Arbazacius, } \\
\text { comes } \\
\text { (Isauriae?) } \\
\end{array}$ & $\begin{array}{l}\text { Zos. V 25; } \\
\text { Marc. Com. s.a. } \\
405 .\end{array}$ & $\%$ & $\begin{array}{l}\text { WooDs (1998) 114; } \\
\text { nach PLRE II 127f, „? } \\
\text { comes rei militaris". }\end{array}$ \\
\hline (434) & $\begin{array}{l}\text { Longinus, } \\
\text { nicht comes } \\
\text { Isauriae, } \\
\text { sondern wohl } \\
\text { comes rei } \\
\text { militaris } \\
\end{array}$ & $A C O$ I 4 p. 87. & $\%$ & $\begin{array}{l}\text { PLRE II } 687 \text { s.n. } \\
\text { 'Longinus 1', } \\
\text { „probably comes et } \\
\text { praeses Isauriae" }\end{array}$ \\
\hline
\end{tabular}




\begin{tabular}{|c|c|c|c|c|}
\hline$(473)$ & Illus & $\begin{array}{l}\text { Patria Const. } \\
\text { III } 33 \text { p. } 227 .\end{array}$ & $\begin{array}{l}\text { Repertorium 3 I2 'Lam } \\
\text { 3' = BENT (1891) 259, } \\
\text { Nr. 32; HEBERDEY / } \\
\text { WILHELM (1896) 50; } \\
\text { SEG XXXVI } 1240\end{array}$ & $\begin{array}{l}\text { LAMINGER-PASCHER } \\
\text { (1974b) 67; HUNGER } \\
\text { (1986); DAGRON / } \\
\text { FEISSEL (1987) 54; } \\
\text { FEISSEL (1999); } \\
\text { ELTON (2000) 407. }\end{array}$ \\
\hline 479 & \begin{tabular}{|l|} 
Aetius, \\
comes \\
Isauriae (?) \\
\end{tabular} & $\begin{array}{l}\text { Joh. Ant. frg. } \\
211,4 \text {. }\end{array}$ & $\%$ & $\begin{array}{l}P L R E \text { II } 20 \text { s.n. } \\
\text { 'Aetius 4'. }\end{array}$ \\
\hline $\begin{array}{l}488-(?) \\
491\end{array}$ & \begin{tabular}{|l|} 
Lilingis, \\
comes et (?) \\
praeses \\
Isauriae
\end{tabular} & $\begin{array}{l}\text { Mal. XV } 14 \text { p. } \\
389 ; \\
\text { Theoph. AM } \\
5985 ; \\
\text { Suda B } 279 . \\
\end{array}$ & $\%$ & $\begin{array}{l}\text { PLRE II 683f., s.n. } \\
\text { 'Lilingis'. }\end{array}$ \\
\hline
\end{tabular}

